\title{
DATA MANAGEMENT PLATFORM SUPPORTING VOLUNTEER OCCUPATION SERVICES
}

\author{
Damien Nicolas, Adnan Emeri, Marie Laure Watrinet and \\ Djamel Khadraoui \\ LIST: Luxembourg Institute of Science and Technology \\ Maison de l'innovation - 5, Avenue des \\ Hauts-Fourneaux L-4362 Esch-sur-Alzettebourg
}

\begin{abstract}
This paper deals with the Data Management Services, which acts as a link between the User Requirements, the System Requirements and the development of the core IT software components supporting the occupation services performed during the Sponsor project. This gives an overview of the main services provided by the platform, the chosen architecture model including the relationships between the different conceptual models and the data models implemented within the platform. As a result, the outcomes of this deliverable will constitute an input for the development of the platform as a services-oriented platform providing and publishing a complete API for managing organizations, opportunities and volunteers.
\end{abstract}

\section{KEYWORDS}

Data Model, SOA, RESTful Web Services, IT service platform, Ambient Assisted Living, Graph Database, Volunteering Services.

\section{INTRODUCTION}

This paper presents the different services provided by the Sponsor platform. It details the highlevel key systems components and also the data model associated.

The Sponsor architecture design aspires to be scalable, flexible and open. To achieve this goal, it follows the principle of Service-Oriented Architecture (SOA) design. Thus, the system is component-based and provides some flexibility in deployment.

The structure of this paper is the following: the first section presents briefly the overall architecture of the platform and its data model associated; then the second section describes the core services providing support to the targeted entities: organizations and volunteers. Finally, the third section gives the description of common services such as registration, search or communication services. 


\section{ARCHITECTURE AND DATA MODEL}

The architecture of the Sponsor platform is based on the SOA approach, facilitating the flexibility, scalability and openness properties of the platform

\section{A. SOA APPROACH OVERVIEW}

The Sponsor platform shall satisfy the targeted requirements by interconnecting different internal components or external systems. Therefore, the most suitable architectural design seems to be a Service-Oriented Architecture (SOA) design that is a modular and open design. The Sponsor architecture is designed and implemented on basis of these principles where SOA is considered as an evolution of any distributed computing, putting the focus on the modularity and the interconnection of the systems from a Service point of view.

Further underlying and enabling ingredient is given by the metadata which are sufficient to describe not only the characteristics of these services, but also the data that implement them. XML has been used extensively in SOA to create data which are wrapped in a nearly exhaustive description container. Analogously, the services themselves are typically programmed in WSDL (Web Services Description Language), and communication protocols in SOAP, that is a protocol for exchanging XML-based messages over computer networks, by using normally HTTP/HTTPS. In the scope of the project, a different approach is adopted for specifying the communication format and the communications protocol:

- For the communication between the front-end and the back-end, the JSON language was selected, which is less verbose than XML and hence well adapted to other languages for the communication between two systems;

- For the communications protocol, a RESTful approach was chosen because of its simplicity (regarding the SOAP protocol) and the way it manages the access of resources through the HTTP/S operations such as GET, POST, DELETE or PATCH

Sponsor architecture is based on services on top of centralized system called here as a back-end, and on events that will be the main way of communication between the components.

The rationale behind the choice of a SOA design are motivated by the following:

- The ability to reuse developed components, to enable sharing modules between applications and inter application changes;

- The flexibility of enriching the Sponsor system with new components to satisfy possible new business needs;

- The openness and interoperability by design, in order to share components between platforms and environments

- The distribution enabled by the ability to deploy remotely some components and/or an associated data storage;

- Scalability by design 


\section{B. LAYERS DESCRIPTION}

A system architecture is generally organized in different layers, also known as the n-tiers architecture pattern [1], a fact that enables a logical cut of the targeted architecture with specific responsibilities assigned to every layer. Each layer can be also split into sub-layers if needed. This approach offers the main advantage of facilitating on the one hand the conception and the development of the platform, whereas on the other hand it ensures a better maintainability of the system in the future. The following schema (Figure 1) provides an overview of the layers of the SpONSOR architecture.

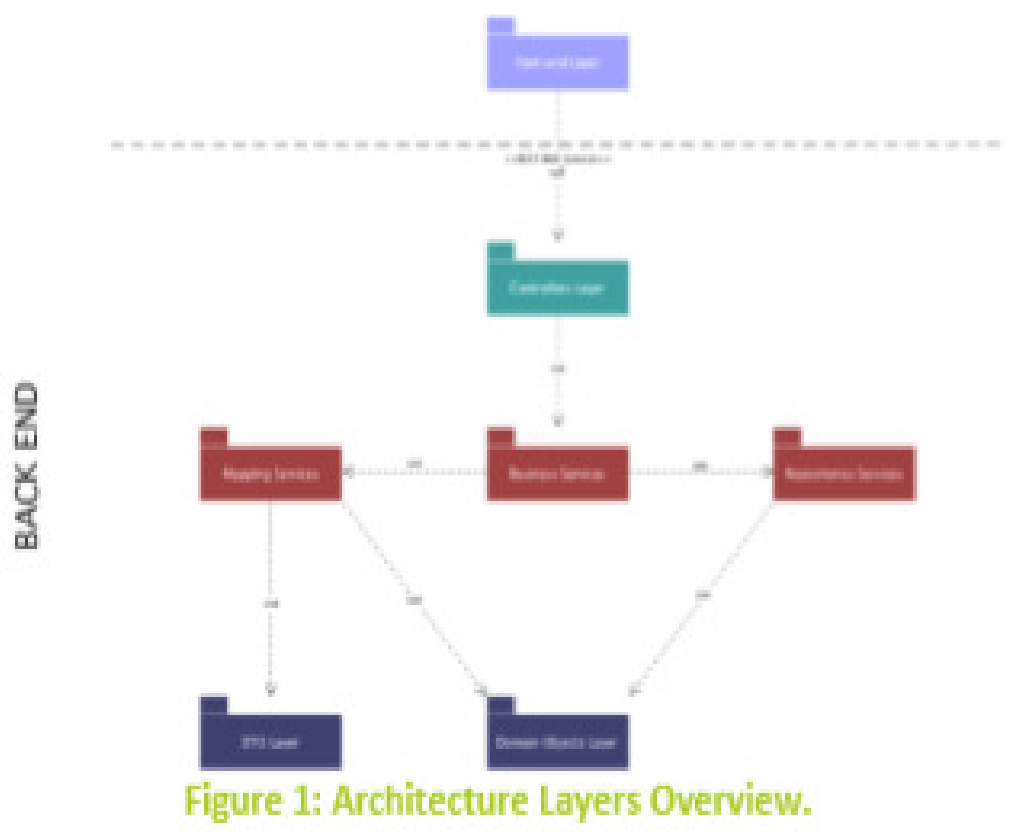

Each layer has a specific rationale and purpose:

- Front-end layer: this layer contains all the graphical user interface elements, such as web pages (written using the dedicated format HTML5), web-oriented languages such as Javascript, styles rules with CSS3, and some logic related to the navigation of the user and the display of the data..

- Controllers Layer: this layer contains the interfaces (endpoints) provided by the SpONSOR backend. This is the main and only entry point for accessing provided services. These entry points supports RESTful web services and uses the JSON format as the format for input and output communications between the front-end and the back-end.

- Business Services: this layer handles the business services of the SpONSOR platform. Each business service is grouped in a dedicated package, in order to facilitate the maintainability of the platform. This layer is the only layer used by the Controllers one, which enables in turn 
- Mapping Services: this layer performs the mapping between the objects of the Domain layer and the objects of the Data Transfer Objects (DTO) layer. In order to ensure the encapsulation and the handling of possible changes for the services at a minimized cost, objects of the Domain are not directly exposed to the interfaces. Only the DTO layer is visible and used from the front-end layer.

- Repository Services: this layer manages the storage and the retrieval of the data from and to the database, used by the platform. This layer is based on ACID [2] transactions. This layer can also be replaced by another one if the database system should be changed, without any additional costs,

- Data Transfer Object Layer: this layer follows the well-known DTO pattern [3] to hide the real objects manipulated by the platform. A DTO is an object that aggregates some data from the data layer domain, in order to present meaningful objects to the requester, and to minimize the network connections between the requester and the provider

- Domain Objects Layer: this layer contains the core data model manipulated by the SpONSOR platform. By design, this layer has no dependencies with the other layers

The Figure 1 provides also the interactions, and therefore the dependencies, between the layers of the architecture.

\section{DATA MODEL OVERVIEW}

This section gives a description of the data model used by the platform. From a bird-view perspective, three concepts are mandatory for the SpONSOR platform:

- Organization;

- Volunteer;

- Opportunity

An organization is an entity comprising multiple people, such as an institution or an association, which has a collective objective and is linked to an external environment. In our case, an organization is senior-supportive and must be involved in the well-being of the senior persons with a clear mission of supporting activities of seniors

A volunteer is a person who wishes to provide services, while renouncing to any financial gain, to benefit of another person, group or association. In our case, a volunteer can be anyone who is registered, as a such, in the platform.

An opportunity is defined as a time when a particular situation makes it possible to do or achieve something. In our case, an opportunity is a task (recurrent or not) provided by an organization, that requires one or more volunteers to be achieved.

The core relations between these three concepts are the following: 


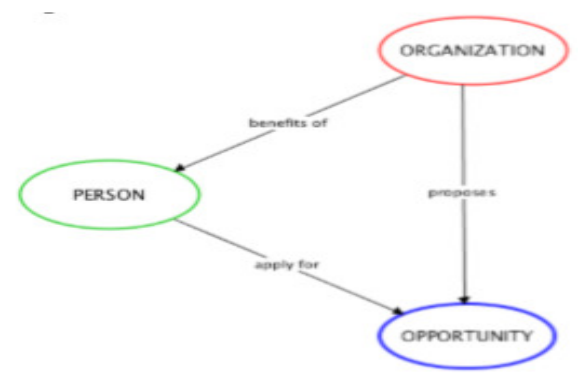

Figure 2: Core Concepts model.

An organization proposes an opportunity. A person can apply to this opportunity. If he/she fulfils all or a part of the requirements needed by this opportunity, he/she can become a volunteer if the organization agrees his/her application and then the organization can benefit of his/her skills and time

The model shown above is a simplified version of the one used by the SpONSOR platform. As shown in the Figure 4, the SpONSOR model considers additional information such as:

- Location of the opportunity (and person)

- Time constraints

- Driving Licenses

- Professional competencies

- Experience

- Type (hard, soft) and categories of skills (technical, management, organizational, etc.)

- Profiles of the eventual beneficiary of the opportunity in case of home care tasks.

The schema (Figure 3) is an excerpt of the data model of the SpONSOR platform. The data model of the SpONSOR platform is implemented through a graph-oriented database, that means a concept (such as a Person, an Opportunity, and so on) is a node and the relation "apply for" is represented by an edge between a Person and an Opportunity, and can have several properties.

This approach has several advantages:

- There is no schema for the database: the nodes and the edges (relationships) can be created at the runtime, a fact that offers a very good flexibility to handle new concepts;

- The scalability is very good, because of the nature of the graph. The graph traversal, to be performed for instance for a search, is not depending on the number of nodes;

- Some relations are naturally modeled with a graph, as for instance the relation between an organization and its volunteers

The following classes (or concepts) with theirs associated properties (that are not shown below) are implemented in the data model:

- Person: represents a human person, that can be a volunteer; 
- Organization: represents a logical group of persons with the same collective goal;

- Organization type: represents the type (legal view) of an organization;

- Organization category: represents the category (humanitarian, social, etc.) of an organization;

- Opportunity: represents a time where something can be done by a person;

- Opportunity type: represents the type of the opportunity (home care, accompanying seniors, etc.)

- Availability: free time available to perform an opportunity (for a person);

- Location: GPS coordinates (in numeric format) of an address;

- Address (including zip code, street, state, city and country);

- Gender: represents the fact of being male or female for a person;

- Title: represents the status of a person to show his/her rank or profession (Mr., Miss, Dr., etc.);

- Civil status: represents the various distinct options that describe a person's relationship with a significant other person (married, single, divorced, etc.);

- Occupation: represents the job or the profession of a person;

- Professional competencies: represents the skills or competences delivered by an official institute, administration, school or university (diploma, certificate, etc.); Experience: represents a non-professional related competence that can be valorized. An example of experience can be a first activity as a helper for an association, without being validated by a diploma. This field should contain such kind of experience, while qualifying experience should be mentioned into the "professional competencies";

- Nationality: represents the nationality of a person

- Time commitment: represents the time constraints (date and time required) of an opportunity;

- Document (such as avatar, organization logo and CV);

- Driving license: represents the driving licenses that a person can have;

- Spoken language: represents the spoken languages (with indication of the language mastering level) of a person;

- Event: represents an event organized or sponsored by an organization;

- Questionnaire: represents a list of questions that a person should answer, according to some rules defined by the organization. It is also used for representing General Condition(s) of an Organization.

- Question (including type of expected answers: binary, single or multiple answers, free text): represents a question and its possible answer type that a person should answer, according some rules defined by the organization;

- Sector of activity (for an organization): represents the sector(s) of activity of an organization;

- Recipient (also known as the beneficiary of an opportunity);

- Religion: represents the religion of the person;

- Video: a person can have one or several video sequences linked to his/her profile

- Skill: represents a skill; $\square$ Skill type: represents the type of skill (soft or hard)

- Skill category: represents the category of the skill (technical, organizational, etc.);

- Interest: represents an interest for a person;

- Training: represents a set of videos that an organization can manage for a training purpose 
All these concepts are implemented as a Node, and the relationships between these concepts are implemented as Edges, with specific properties when needed.

\section{CORE SERVICES PROVIDED BY THE PlatForm}

This section presents the main services provided by the Sponsor platform for the two targeted entities: namely organization and volunteer. First let's give a definition of a service:

A service is an action executed by a provider for a

requester.

As the communication between the front-end (also known as the Graphical User Interface) and the back-end used only web services (and specifically REST web services), an action is typically performed by executing some fragments of a program [4]. The interaction between the two actors (provider and requester) is realized through controllers that exposed specific endpoints formalized by one or several interfaces

An interface defines the supported processing in the form of an input message, an output message, and its URI to use, by providing the protocol to use (HTTP, HTTPS, FTP, etc.) for instance.

Different types of services could be found in the literature:

- Applicative service: service about a particular business domain of the company

- Functional service: service identified in the functional requirements phase

- Technical service: service that serves a specific technical process such as storage data into a database. Different levels of services can also be specified:

- High-level service: allows the access to high-level functionalities of the Business Services (high granularity)

- Low-level service: enables the access to low-level or basics functionalities of the Business Services (low granularity)

\section{A. SPONSOR PLATFORM SERVICES}

By definition, a Service-Oriented Architecture is an architecture that exposes its services in the form of independent and well-defined services. Some services are autonomous and some services can use other services (by collaboration, synchronization or composition) to provide further services with strong added value. In addition, these services are also carried out with ready-to-use interfaces

Considering the set of functional requirements, the following high-level services were developed within the Sponsor platform: 
- Common Services o Registration

○ Login - Logout

○ Communication

- Search

○ Statistics

- Organisation-related Services

- Organisation Management

- Profile management

- Event management

- Panel management

- Opportunity Management

o Training Management

- Volunteer-related Services o Volunteer Management

- Profile management

- Legal \& Ethical advisor

The following schema gives an overview of the services supported by the platform:

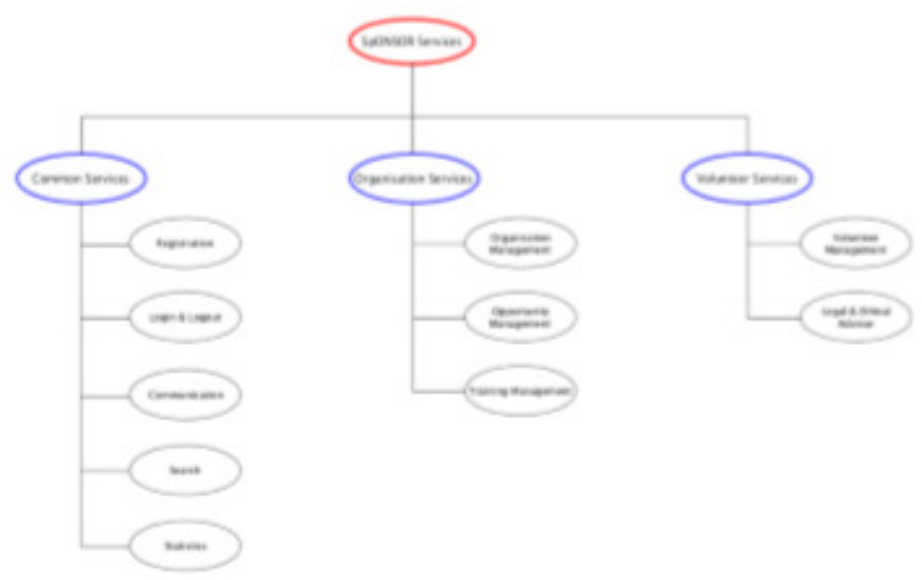

Figure 3: Sponsor Services Overview

Each service is briefly described in the following sections.

\begin{tabular}{|l|}
\hline Name of service: Organization Profile Management starts always by /organization \\
\hline Objective(s): this service supports all the operations related to the management of an \\
organization, including the management of its own profile \\
\hline $\begin{array}{l}\text { Description: an organization needs to support some business operations such as the } \\
\text { management of its profile, including adding, removing, updating its logo, its own } \\
\text { documents, and its general conditions if needed. }\end{array}$ \\
\hline $\begin{array}{l}\text { Functionalities: Profile creation; Profile update; Profile deletion; Document addition; } \\
\text { Document deletion; General condition addition; General condition deletion; General } \\
\text { condition update; Logo addition/update; Logo deletion; Get the creator of an } \\
\text { organization. }\end{array}$ \\
\hline
\end{tabular}


The following service manages the events proposed by a given organization

\begin{tabular}{|l|}
\hline $\begin{array}{l}\text { Name of service: Organization Events Management starts always by } \\
\text { /organization/\{organization ID\}/event. }\end{array}$ \\
\hline $\begin{array}{l}\text { Objective(s): this service supports all the operations related to the management of the } \\
\text { events proposed by an organization }\end{array}$ \\
\hline $\begin{array}{l}\text { Description: an organization can provide information on some events related to its } \\
\text { business or located near the organization. }\end{array}$ \\
\hline Functionalities: Event creation; Event deletion; Event update; Get an event. \\
\hline
\end{tabular}

The following service managed the panel of potential volunteers for a given organization:

\begin{tabular}{|l|}
\hline $\begin{array}{l}\text { Name of service: Organization Panel Management starts always by } \\
\text { /organization/\{organizationID\}/panel }\end{array}$ \\
\hline $\begin{array}{l}\text { Objective(s): this service supports all the operations related to the management of the } \\
\text { panel of an organization }\end{array}$ \\
\hline $\begin{array}{l}\text { Description: an organization can manage into a "virtual" panel a list of potential } \\
\text { volunteers. These volunteers could be contacted by the organization later if needed. }\end{array}$ \\
\hline $\begin{array}{l}\text { Functionalities: Volunteer addition on a panel; Volunteer deletion from a panel; } \\
\text { Organization panel deletion; Get all volunteers from a panel managed by an } \\
\text { organization }\end{array}$ \\
\hline
\end{tabular}

The following services are helper services related to one or all organizations

Name of service: Organization Helper Service starts always by /organization/ Objective(s): this service provides some operations that perform basic searches on the database related to organization(s).

Description: the organization helper service frames basic searches related to information managed by organization, such as all events or all organizations Functionalities: Get all events with criteria; Get all organizations; Get all organizations sorted by countries.

\section{OPPORTUNITY RELATED SERVICES}

The following section gives the services offered to manage an opportunity.

\begin{tabular}{|l|}
\hline Name of service: Opportunity Service starts always by /opportunity/ \\
\hline $\begin{array}{l}\text { Objective(s): this service provides basic operations to manage opportunities from the } \\
\text { organization point of view }\end{array}$ \\
\hline $\begin{array}{l}\text { Description: this service provides support for the management of opportunity by giving } \\
\text { the essentials functionalities }\end{array}$ \\
\hline $\begin{array}{l}\text { Functionalities: Opportunity creation; Opportunity deletion; Opportunity update; Get an } \\
\text { opportunity. }\end{array}$ \\
\hline
\end{tabular}

Name of service: Opportunity Helper Service starts always by /opportunity Objective(s): this service provides search functionalities on opportunities. Description: search functionalities such as getting all opportunities or only a subset with some criteria are provided by this service.

Functionalities: Get all opportunities; Get opportunities with criteria. 


\section{VOLUNTEER- RELATED SERVICES}

The following services provides support for the management of volunteers:

\begin{tabular}{l}
\hline Name of service: Person Profile Management starts always by /person \\
\hline $\begin{array}{l}\text { Objective(s): this service supports all the operations related to the management of a } \\
\text { person, including the management of its own profile }\end{array}$ \\
\hline $\begin{array}{l}\text { Description: a person needs to support some business operations such as the } \\
\text { management of its profile, including adding, removing, updating his/her avatar, and } \\
\text { his/her documents if needed }\end{array}$ \\
\hline $\begin{array}{l}\text { Functionalities: Profile creation; Profile update; Profile deletion; Get a profile; } \\
\text { Document addition; Document deletion; Avatar addition/update; Avatar deletion }\end{array}$ \\
$\begin{array}{l}\text { Name of service: Person Events Management Service starts always by } \\
\text { /person/\{personID\}/event }\end{array}$ \\
\hline $\begin{array}{l}\text { Objective(s): this service supports all the operations related to the management of the } \\
\text { events that a person could be interested in }\end{array}$ \\
\hline $\begin{array}{l}\text { Description: a person can register (or unregister) himself/herself for an event proposed } \\
\text { by one or several organizations }\end{array}$ \\
\hline Functionalities: Event registration; Event unregistration \\
\hline Name of service: Person Helper Service starts always by /person/ \\
\hline $\begin{array}{l}\text { Objective(s): this service provides search functionalities on volunteers or information } \\
\text { related to the volunteers }\end{array}$ \\
\hline $\begin{array}{l}\text { Description: search functionalities such as getting all volunteers or only a subset with } \\
\text { some criteria are provided by this service, setting or getting preferred organizations or } \\
\text { opportunities for a person, etc. }\end{array}$ \\
\hline $\begin{array}{l}\text { Functionalities: Get all persons; Get events for which a person is registered; Get } \\
\text { preferred organizations; Get preferred opportunities; Set/update preferred organizations; } \\
\text { Set/update the preferred opportunities; Remove preferred organizations; Remove } \\
\text { preferred opportunities. }\end{array}$ \\
\hline
\end{tabular}

\section{E. MANAGEMENT SERVICES}

Name of service: Management Service starts always by /management/

Objective(s): this service provides specific management functionalities for organization and volunteer

Description: this main service provided several services to manage the application status from the both side: volunteer and organization. A person can apply to an opportunity, withdraw an application or be interested by opportunities and organizations. An organization can set the status of the volunteer: ACCEPTED, REFUSED, STANDBY, CONVOKED INTERVIEW, CONVOKED TRAINING, QH. Functionalities: Get opportunities posted by an organization; Get opportunities status for a volunteer; Get opportunities for a volunteer; Get organizations that posted an opportunity; Get persons with specific status related to an opportunity; Get status for a volunteer; Get volunteer status for an opportunity; Get applicants for opportunities; Set application status apply (APPLY) for an opportunity; Set application status withdraw (WITHDRAW) for an opportunity; Set the general status for an application for a volunteer; Set the status for a volunteer. 
Some business rules are also implemented for some services. For example, a volunteer can only withdraw his application once the application has indeed been submitted. When a volunteer wants to withdraw his/her application, however before having filed it, an error 409 is returned by the platform with an appropriate message.

\section{COMMON SERVICES}

This section gives an overview of the common services provided by the platform. The term "common services" refers to services used by all the entities managed by the platform.

\section{A. REGISTRATION AND LOGIN SERVICES}

These services provide registration and login into the platform.

\begin{tabular}{|c|}
\hline $\begin{array}{l}\text { Name of service: Registration and Login Service starts by /register for registration starts } \\
\text { by /login for login }\end{array}$ \\
\hline $\begin{array}{l}\text { Objective(s): this service enables the registration and the login to the platform. In } \\
\text { addition, two services are provided: } \\
\text { - Change the password: a user can change his/her password } \\
\text { - Reset the password: reset the password if the user forgets it }\end{array}$ \\
\hline $\begin{array}{l}\text { Description: an organization can provide information on some events related to its } \\
\text { business or located next to the organization. }\end{array}$ \\
\hline $\begin{array}{l}\text { Functionalities: Registration of a person; Registration of a representative of an } \\
\text { organization; Log in into the platform; Reset the password; Change the password }\end{array}$ \\
\hline
\end{tabular}

\section{B. COMMUNICATION SERVICES}

This service provides communication means between the volunteers and the organizations. The communication is realized through messages, and email notifications.

\begin{tabular}{|c|}
\hline $\begin{array}{l}\text { Name of service: Communication Service starts by /message for communication } \\
\text { Objective(s): this service enables sending and receiving messages between volunteers } \\
\text { and organizations. }\end{array}$ \\
\hline $\begin{array}{l}\text { Description: volunteers and organizations can communicate through an internal } \\
\text { messaging channel. A message basically corresponds to an email including the } \\
\text { following data: } \\
\text { - Sender } \\
\text { - Receiver(s): can be an email address } \\
\text { - Subject } \\
\text { - Content Depending on some actions, emails are also sent automatically by the } \\
\text { platform. }\end{array}$ \\
\hline $\begin{array}{l}\text { Functionalities: Get all messages received or sent by an organization; Get all messages } \\
\text { received or sent by a person; Get a message by its unique identifier; Create and post a } \\
\text { message to one or several receivers, sent by an organization; ; Create and post a } \\
\text { message to one or several receivers, sent by a volunteer; Delete a message, identified by } \\
\text { its unique identifier }\end{array}$ \\
\hline
\end{tabular}




\section{SEARCH SERVICES}

The following services provides search functionalities, limited to some elements of the model such as:

- Opportunity

- Organization

- Event

\begin{tabular}{|c|}
\hline Name of service: Search Service \\
\hline $\begin{array}{l}\text { (s): this service provides search functionalities for the following elements: } \\
\text { ity, organization and event }\end{array}$ \\
\hline $\begin{array}{l}\text { Description: volunteer and organization would search information on opportunities, } \\
\text { organizations and events stored in the database. In order to provide such functionalities, } \\
\text { a full-text search is made with the help of a dedicated search engine: the elastic search } \\
\text { that provides full-text search with an indexing mechanism to speed-up the search. }\end{array}$ \\
\hline Functionalities: Get all opportunities that contain \\
\hline
\end{tabular}

The elastic search database is populated/updated each time when an opportunity, an organization or an event is created, respectively updated. These actions are totally transparent for the user, and are made asynchronously to avoid blocking the graphical user interface. The search and matching functionalities are note very detailed in this paper.

\section{STATISTICS SERVICES}

Several statistics services are also provided by the platform. The statistics module makes calculations based on events and opportunities indexed for searched, and offers an additional service to index person profiles. The following statistics are available:

- Most frequent terms in opportunities

- Most searched terms in events and opportunities

- Most frequent interests and most frequent skills appearing in the profiles.

\begin{tabular}{|c|}
\hline Name of service: Statistics Service \\
\hline $\begin{array}{l}\text { Objective(s): this service provides statistics for the following elements: opportunity, } \\
\text { skills, interests and events }\end{array}$ \\
\hline $\begin{array}{l}\text { Description: it could be interesting to know the most frequent terms used in the } \\
\text { description of opportunities for a volunteer, in order to know if one of his/her skills or } \\
\text { interests is frequently occurring or searched, for example. This service furnishes also } \\
\text { other useful statistical data both for an organization (regarding the most frequent terms } \\
\text { appearing in person profiles, for example) and for a person (most frequent terms in } \\
\text { opportunities profile) }\end{array}$ \\
\hline $\begin{array}{l}\text { Functionalities: Get most frequent terms in opportunities; Get searched items in events; } \\
\text { Get searched items in opportunities; Get most frequent interests in profiles; Get most } \\
\text { frequent skills in profiles; Get legal document(s) that contain specific words. }\end{array}$ \\
\hline
\end{tabular}

By default, the statistics are calculated by taking into account the data collected over the previous six months. This service is also responsible for indexing legal documents managed by the legal service. Once legal documents are indexed, it is possible to perform a full-text search on them. 


\section{E. LEGAL SERVICES}

The legal service provided by the platform gives some advices to the volunteer on the legal aspects related to the opportunity he/she wants to apply for. The mechanism is based on a Legal Tree approach [5]. This service manages legal documents with the following model.

\begin{tabular}{|l|l|l|l|}
\hline Field & Type & Description & Example value \\
\hline id & String & $\begin{array}{l}\text { Technical field. } \\
\text { Automatically } \\
\text { generated when a } \\
\text { document in inserted. }\end{array}$ & Mf69875 \\
\hline Name & String the & $\begin{array}{l}\text { Loi fédérale sur } \\
\text { l'assurancevieillesse } \\
\text { et survivants }\end{array}$ \\
\hline Lang & String & $\begin{array}{l}\text { Name of } \\
\text { document. }\end{array}$ & $\begin{array}{l}\text { (Optional) } \\
\text { language of the } \\
\text { document. }\end{array}$ \\
\hline Sections & $\begin{array}{l}\text { Array of Section } \\
\text { object }\end{array}$ & $\begin{array}{l}\text { Thections that compose the document. } \\
\text { This field is automatically managed by the } \\
\text { system }\end{array}$ \\
\hline Pages & Integer & $\begin{array}{l}\text { (Optional) The } \\
\text { number of pages of } \\
\text { the document if } \\
\text { applicable. }\end{array}$ \\
\hline Ref & String & $\begin{array}{l}\text { The file name if the } \\
\text { document is a file, } \\
\text { or the URL if the } \\
\text { document is a web } \\
\text { page. }\end{array}$ & \\
\hline
\end{tabular}

A section is a concept that is managed by the system when indexing the content of the legal document. The associated object has the following schema:

\begin{tabular}{|l|l|l|l|}
\hline Field & Type & Description & Example value \\
\hline Name & String & Name of the section & Article 7 \\
\hline cont. & String & $\begin{array}{l}\text { Content of the } \\
\text { section }\end{array}$ & $\begin{array}{l}\text { Le Conseil fédéral } \\
\text { peut fixer }\end{array}$ \\
\hline Page & Int. & $\begin{array}{l}\text { The page on which } \\
\text { the section starts, if } \\
\text { applicable }\end{array}$ & \\
\hline
\end{tabular}

\section{CONCLUSION}

This paper presented the data management services developed in the SpONSOR platform, that are in line with the User Requirements \& System requirements, the scenarios developed as well as with the legal guidance and requirements.

The main outcomes of this paper are: 
- Identification and definition of the major SpONSOR services that are implemented in the platform.

- Definition of the main interactions between the different layers of the architecture.

Furthermore, this paper aimed at establishing a bridge between conceptual developments and results that were achieved in requirements phase, and the implementation phase, in which the Sponsor platform and its associated services are being developed.

We acknowledge that the work presented in this paper is composed of part of the technical contribution (Data Management Model) developed under an AAL [6] funded project (SPONSOR [7] [8]) and supported by the National Research Fund of Luxembourg

\section{REFERENCES}

[1] https://msdn.microsoft.com/enus/library/ee658117.aspx\#NTier3TierStyle

[2] Haerder, T.; Reuter, A. (1983). "Principles of transaction-oriented database recovery". ACM Computing Surveys. 15 (4): 287. doi:10.1145/289.291

[3] https://martinfowler.com/eaaCatalog/dataTransferObject.html.

[4] https://www.w3.org/TR/ws-arch/\#action (section Explanation).

[5] Adnan Imeri, Abdelaziz Khadraoui, Thang Le Dinh, Djamel Khadraoui: Personalization of Legal and Ethical Information in ICT Platforms: The Approach of Legal Decision Tree. Computer and Information Science 10(1): 77-88 (2017).

[6] http://www.aal-europe.eu/

[7] Amedeo Cesta, Gabriella Cortellessa, Riccardo De Benedictis, Francesca Fracasso, Daniel Baumann, Stefano Cuomo, Julie Doyle, Adnan Imeri, Djamel Khadraoui, Pierre Rossel: Personalizing Support to Older Adults who Look for a Job with the SpONSOR Platform. AI*AAL@AI*IA 2016: 105-122

[8] http://www.sponsor-aal.eu 


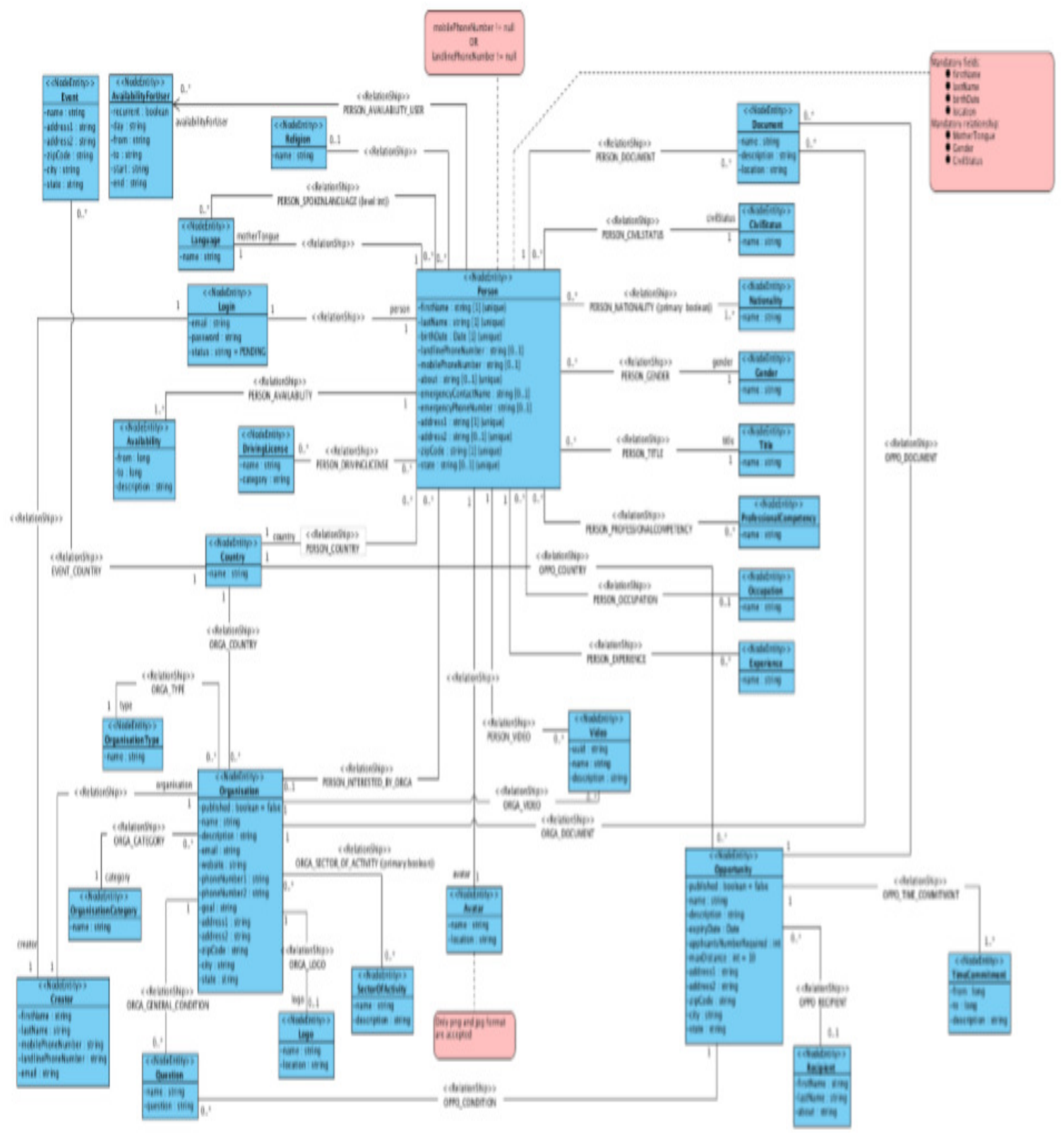

Figure 4: Data model (extract). 